\title{
PROBING NON-ADHERENCE TO PRESCRIBED MEDICINES? A BIVARIATE DISTRIBUTION WITH INFORMATION NUCLEUS CLARIFIES
}

\author{
Ramalingam Shanmugam \\ School of Health Administration, Texas State University, TX 78666, USA
}

Received 2014-06-13; Revised 2014-06-17; Accepted 2014-07-17

\begin{abstract}
No illness gets cured without the patient's adherence to the prescribed medicine (s). Reasons such as too many medicines, lack of health insurance coverage, high co-payment cost, loss of cognitive memory to take. are commonly noticed for non-adherence. In some illnesses, the patients who do not adhere to the prescribed medicines end up again in hospital. How should the pertinent data be analyzed to learn? Currently, there is no suitable methodology to scrutinize the data for a clear assessment about the significance of a reason. To fulfil such a need, this article develops and demonstrates a new underlying bivariate probability model for the data and a statistical methodology to extract pertinent information to check whether the non-adherent proportion of patients to medicine (s) is significant enough to come up with strict remedial policies. To start with, the case of too many prescribed medicines is examined. Then, the repeated hospitalization due to nonadherence is examined. The contents of this article could be easily extended to other reasons of nonadherence as well. In the presence of a reason, there might exist a number of non-adherent $X$ and a number of adherent, $Y$ patients. Both $X$ and $Y$ is observable in a sample of size $n_{1}$ with the presence of a reason and in another random sample of size $n_{2}$ with the absence of a reason. The total sample size is $n=n_{1}+n_{2}$. Let $0<\phi<1$ and $0<\rho<1$ denote respectively the probability for a reason to exist in a patient and the probability for a patient to be non-adherent to the prescribed medicines. Of interest to the medical community is the trend of the sum, $T=X+Y$ and $Z=n-X-Y$ denoting respectively the total number of non-adherent and adherent patients irrespective of a reason. Hence, this article constructs a bivariate probability distribution for $T$ and $Z$ utilize it to explain several non-trivialities. To illustrate, non-adherence patients' data in the literature are considered. Because the bivariate probability distribution is not seen in the literature, it is named as non-adherent bivariate distribution. Various statistical properties of the non-adherent bivariate distribution are identified and explained. An information based hypothesis testing procedure is devised to check whether an estimate of the parameter, $\rho$ is significant. Two closely connected factors for the patients not adhering to the prescribed medicines are examined. The first is a precursor and it is that too many medicines are prescribed to take. In an illustration for the first reason, the probability for a patient not to adhere the medicines is estimated to be 0.78 which is statistically significant. The second is the post cursor and it is that the patients not-adhering to the medicines are more often hospitalized again. In an illustration of the second factor, the probability for the diabetic patients not to adhere the medicines is estimated to be 0.44 which is significant. The statistical power of accepting the true non-adherence probability by our methodology is excellent in both illustrations. A few comments are made about the future research work. Other reasons for the patients' non-adherence might exist and they should also be examined. A regression type prediction model can be constructed if additional data on covariates are available. A principal component analysis might reveal clusters of reasons along with the grouping of illnesses if such multivariate data become available. The usual principal component analysis requires bivariate normally distributed data. For the data governed by the non-adherent bivariate distribution, a new principal component
\end{abstract}


methodology needs to be devised and it will be done in a future research article. The contents of this article is the conceptual foundation for such future research work.

Keywords: Healthcare Cost Saving, Hospital Readmission Rate, Correlation, Conditional Mean and Variance, Information, p-Value, Statistical Power

\section{MOTIVATION}

A concern to the medical community, families of the patients, hospital management, insurance industries, healthcare agencies is the non-adherence of patients to the prescribed medicines. If the medicines are not taken in accordance with the prescription, it guarantees no complete or delayed cure. In some illnesses such as cardiac vascular illness, the patients may have to be readmitted in the hospital and it increases the healthcare cost. There may be many reasons for the patients not taking the prescribed medicines as advised by the physicians. When the patients are elderly, have diminished memory, or possess cognitive inability to adhere the medicines, they are in need of support members in the family or social networks but the support persons might be busy with their job or far away on a tour. The patients' procrastination might a reason. The health insurance may not cover the medicine cost or the co-payment for the medicine might be not affordable. More important reason might be that too many medicines are prescribed. The over-medication is not uncommon but is inappropriate practice by some physicians and this practice has been challenged. The over medication may happen because the physician is unaware of other medications the patient is taking, physician's human error, or an excessive profit motives on the part of physicians for the pharmaceutical industry. In the illustrations, this article focuses on the over-medication and the hospitalization again versus nonadherence to medicines by the patients.

How the data on the number of prescribed medicines versus the number of non-adherent patients be analyzed and interpreted? A search of the literature reveals that an appropriate probabilistic conceptual framework or statistical technique does not exist. While the theme is of high practical importance, it is time to develop a methodology to fulfill the need and it is done in this article. Bivariate distributions have been of interest to the data analysts. For an example, (Teamah and Ahmed's 2009) illustration of bivariate exponential distributions. From the basics, a new bivariate probability distribution is created and its statistical properties are established to understand whether a significant proportion of patients exists not adhering to the prescribed medicines for a stated reason. The findings will be useful to the medical professionals, patients' relatives, the healthcare insurance industries among others.

\section{NEW BIVARIARE DISTRIBUTIONS}

To be specific, let $0<\phi<1$ and $0<\rho<1$ denote respectively an unknown probability that a patient is prescribed with less number of medicines and an unknown probability that a patient adheres to the prescribed medicines. Four mutually exclusive outcomes are possible with respect to these two aspects. They are: (1) Over medication and non-adherence with a probability $0<(1-$ $\phi)(1-\rho)<1$, (2) lesser medicines and adherence with a probability $0<\phi \quad \rho<1$, (3) lesser medicines and nonadherence with a probability $0<\phi(1-\rho)<1$ and (4) over medication and adherence with a probability $0<(1-\phi) \rho<1$. Suppose that there are $X, Y$ and $Z=n-X-Y$ number of nonadherent in less-medicated, non-adherent in overmedicated and adherent in both groups in a random sample of size, $n$. The probability trend of the random variables: $X$ and $Y$ is a bivariate distribution Equation 1:

$$
\begin{aligned}
& \operatorname{Pr}[X=x, Y=y]=\frac{n !}{x ! y !(n-x-y) !} \rho^{n} \\
& {\left[\phi\left(\frac{1}{\rho}-1\right)\right]^{x}\left[(1-\phi)\left(\frac{1}{\rho}-1\right)\right]^{y}} \\
& 0<\phi<1 ; 0<\rho<1 \\
& x=0,1,2, \ldots, n ; y=0,1,2, \ldots, n .
\end{aligned}
$$

The expression (1) is indeed a bona-fide Probability Mass Function (PMF) since $\operatorname{Pr}[X=x, Y=y]$ is non negative and sum to one over the sample space of $x$ and $y$. Marginally viewing, the number, $X$ of overmedicated and adherent follows a probability pattern Equation 2:

$$
\begin{aligned}
& \operatorname{Pr}[X=x]=\frac{n !}{x !(n-x) !} \rho^{n} \\
& {\left[\phi\left(\frac{1}{\rho}-1\right)\right]^{x}\left[\left[\frac{1}{\rho}-\phi\left(\frac{1}{\rho}-1\right)\right]^{n-x}\right.} \\
& 0<\phi<1 ; 0<\rho<1 ; x=0,1,2, \ldots, n
\end{aligned}
$$

Likewise, the number, $Y$ of none over-medicated and adherent follows a probability pattern Equation 3: 


$$
\begin{aligned}
& \operatorname{Pr}[Y=y]=\frac{n !}{y !(n-y) !} \rho^{n} \\
& {\left[(1-\phi)\left(\frac{1}{\rho}-1\right)\right]^{y}\left[1+\phi\left(\frac{1}{\rho}-1\right)\right]^{n-y}} \\
& 0<\phi<1 ; 0<\rho<1 ; y=0,1,2, \ldots, n
\end{aligned}
$$

The expected value and variance of $X$ are $\mu X=n \phi(1-$ $\rho)$ and $\sigma_{X}^{2}=\mu_{X}\left(1-\frac{\mu_{X}}{n}\right)$ which is a concave function of the mean. Likewise, the expected value and variance of $Y$ are $\mu_{Y}=n(1-\phi)(1-\rho)$ and $\sigma_{Y}^{2}=\mu_{Y}\left(1-\frac{\mu_{Y}}{n}\right)$ another concave function of the mean. Consequently, an intrinsic balance exists and it is $\frac{\sigma_{X}^{2}}{\mu_{X}}+\frac{\sigma_{Y}^{2}}{\mu_{Y}}=1+\rho$ which is a plate in a three dimensional graph.

Of real interest to the medical community is indeed the probability pattern of the total number, $Z=X+Y$ of non-adherent and the total number, $Z=n-(X+Y)$ of adherent patients irrespective of the number of prescribed medicines. The bivariate probability pattern of $T$ and $Z$ is obtained with a transformation of variables in (1). That is Equation 4:

$$
\begin{aligned}
& \operatorname{Pr}[Z=z, T=t] \\
& =\frac{n !}{z ! t !(n-z-t) !} \rho^{n-t}(1-\rho)^{n+t-z} ; \\
& 0<\rho<1 ; z=0,1,2, \ldots, n ; t=0,1,2, \ldots, n
\end{aligned}
$$

The expression (4) is a bona fide PMF since $\operatorname{Pr}[Z=$ $z, T=t]$ is non negative and sum to one over the sample space of $z$ and $t$. The PMF in (4) is not found anywhere in the literature and hence, it is named Adherence Bivariate Distribution (ABD) here. Notice that the adherence bivariate distribution is free of the parameter $\phi$ and hence, it is a member of one parameter bivariate family type. The usual Wald's likelihood ratio concept to construct a hypothesis testing procedure is not applicable for a lack of a nuisance parameter. Hence, this article innovatively creates an information concept to construct a hypothesis testing procedure later in the article.

Interestingly, their marginal PMFs are binomial type. That is Equation 5.1 and 5.2:

$$
\begin{aligned}
& \operatorname{Pr}[Z=z]=\frac{n !}{z !(n-z) !} \rho^{z}(1-\rho)^{n-z} ; \\
& 0<\rho<1 ; z=0,1,2, \ldots, n
\end{aligned}
$$

And:

$$
\begin{aligned}
& \operatorname{Pr}[T=t]=\frac{n !}{t !(n-t) !} \rho^{n}\left(\frac{1}{\rho}-1\right)^{t} \\
& 0<\rho<1 ; t=0,1,2, \ldots, n
\end{aligned}
$$

Note that the marginal means are Equation 6.1 and 6.2:

$\mu_{Z}=(n-1)(1-\rho)$,

$\mu_{T}=(n-1) \rho$

But the variances are Equation 6.3 and 6.4:

$\sigma_{Z}^{2}=\mu_{Z}\left[1+\frac{\mu_{Z}}{(n-1)}\right]$

And:

$\sigma_{T}^{2}=\mu_{T}\left[\mu_{T}+\rho\right]$

A regression type projection of one among the two: Total number, $T$ of adherent and $Z$ of non-adherent patients, based on the other requires finding their condition means. The first step in this direction is finding their conditional MPFs and it done next. That is Equation 7:

$$
\begin{aligned}
& \operatorname{Pr}[Z=z \mid T=t]=\left(\begin{array}{c}
n-t \\
z
\end{array}\right)\left(\frac{\rho}{1-\rho}\right)^{z}(1-\rho)^{n-t} \\
& 0<\rho<1 ; z=0,1,2, \ldots, n-t
\end{aligned}
$$

With the conditional mean (regression equation of $Z$ $=z$ for a given $T=t$ ) is $\mu_{z \mid t}=n \rho-\rho t$ with a homosecedacity level $\sigma_{z \mid t}^{2}=\mu_{z \mid t}(1-\rho)$. A lesser homosecedacity means less volatility in the prediction process. The regression results suggest a practical interpretation of the parameter, $\rho$. That is, for an increase of non-adherent patient, one could expect a percent, $0<\rho<1$ decrease of adherent patients with an increased prediction volatility. The converse is similar but opposite. That is Equation 8:

$$
\begin{aligned}
& \operatorname{Pr}[T=t \mid Z=z]=\left(\begin{array}{c}
n-z \\
t
\end{array}\right)\left(\frac{1}{\rho}-1\right)^{t} \rho^{n-z} ; \\
& 0<\rho<1 ; z=0,1,2, \ldots, n-z ;
\end{aligned}
$$


With the conditional mean (regression equation of $T=t$ for a given $Z=z$ ) is $\mu_{t \mid z}=n(1-\rho)-(1-\rho) z$ with a homosecedacity level $\sigma_{t \mid z}^{2}=\mu_{t \mid z} \rho$. For an increase of adherent patient, one could expect a percent, $0<\rho<1$ decrease of non adherent patients with an increased prediction volatility.

Hence, the random variables $Z$ and $T$ must be inversely correlated. The importance of dispersion and the correlations have been demonstrated in Shanmugam (2013b). One wonders what might be their correlation level. Using the PMFs (4), (5) and (6), the correlation, $\operatorname{corr}(Z, T)=\frac{E(Z T)-E(Z) E(T)}{\sqrt{\operatorname{var}(Z) \operatorname{var}(T)}}$ is obtained and it is, after algebraic simplifications, that Equation 9:

$$
\operatorname{corr}(Z, T)=-\sqrt{(1-\rho)\left\{\frac{1}{n}+(1-\rho)\right\}}
$$

where, $n$ is the sample size and $0<\rho<1$ is the unknown probability that a patient would adhere to the prescribed medicines.

Next, we need to estimate the parameters: $\phi, \rho$ For the approach to be useful in practice. The Maximum Likelihood Estimators (MLE) are preferable over others as the MLE are most efficient and optimal (Stuart and Ord, 1994). For this purpose of finding the MLE, consider a random sample $\left(z_{i}, t_{i}\right), i=1,2, \ldots, n$ of size $n \geq$ 2 from the bivariate PMF (4). Differentiating the $\log$ likelihood $1 n \operatorname{Pr}[Z=z, T=t]$ with respect to the parameter $\rho$, equating to zero, its Maximum Likelihood Estimator (MLE) is obtained and it is Equation 10:

$$
\hat{\rho}_{M L E}=\frac{(n-t)}{(n+t)}=\frac{z}{(n+t)}
$$

Likewise, the MLE of the parameter $\phi$, is obtained and it is Equation 11:

$$
\hat{\phi}_{M L E}=\frac{n_{1}}{\left(n_{1}+n_{2}\right)}
$$

Next, a procedure to test the significance of the sample estimate of the adherence parameter, $\rho$ is needed. The usual Wald's (Stuart and Ord, 1994) is not applicable as there is no nuisance parameter in this set up. Hence, there is a necessity to invent another approach to do the hypothesis testing. For this purpose, a new information concept is introduced and utilized in the next section.

\section{NUCLEUS OF INFORMATION ABOUT NON-ADHERENCE IN BIVARIATE DISTRIBUTION}

Information is an interesting concept and it is popularly applied in scientific studies over fifty years. In a seminal article, (Shannon, 1948) introduced an information concept in an attempt to describe the completeness of receiving the sent electronic messages with no distortion. Broadly speaking, Shannon's information is applicable in any stochastic system. In fact, it has been used and appreciated in a variety of fields like satellite communication, medicine, public health, marketing, globalized business, economic, social studies.

However, the Shannon's information is not fault free. The Shannon's information is unnecessarily overloaded in the way it is expressed. The overloading is correctable as it is done in another article by this author. The Shannon's information has been modified and recognized as entropy. The entropy captures disorders in a system of communication. Like the Shannon's information, the entropy concept also has flaws. See the book by (Ben-Naim, 2008) for a catalogue of serious flaws in entropy.

In the past, (Fisher, 1925; Kullback and Leibler, 1951) introduced a different way of capturing information in the data. However, this article amends the Shannon's approach to redefine the information based on what is now named the nucleus in the model connecting the parameters and the observables. Model is an abstraction of the chance oriented reality. Shanmugam (2013a) for an illustration of Poisson model to capture the rareness in a chance oriented reality with respect to the incidences of rape in nations around the world.

A case in point is the bivariate PMF in (4). The nucleus which contains the information in the observables $z$ and $t$ about the parameter $\rho$ is $\rho^{n-t}(1-\rho)^{\mathrm{n}+\mathrm{t}-\mathrm{z}}$, where the component $\frac{n !}{z ! t !(n-z-t) !}$ is void of any relevance and hence is be omitted. Because the logarithm preserves monotonicity of any trend, why not define $\mathfrak{I}_{z, t}=\left(\frac{1}{\rho}-1\right)^{t}\left(\frac{1}{1-\rho}\right)^{z}$, as the information nucleus, ignoring the constants? The expected logarithmic information nucleus is $\mathrm{E}_{z, t}$ (In $\mathfrak{J}_{\mathrm{z}, \mathrm{t}}$ ), where the notation $\mathrm{E}_{z, \mathrm{t}}$ refers the mathematical expectation with respect to the non-adherence bivariate PMF in (4). That is, after simplifications Equation 12: 
$E_{z, t}\left(\ln \mathfrak{I}_{z, t}\right) \approx(n-1) \rho(1-\rho)$

Using (6.2) and (6.3). Additionally, if the expected logarithmic information nucleus is normed in the interval $(0,1)$, it is easy to comprehend its relative importance. For this sake, a normed ratio,

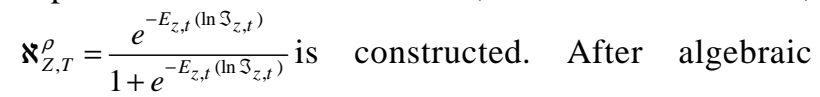
simplifications, it is Equation 13:

$\aleph_{Z, T}^{\rho}=\frac{\rho^{\mu_{T}}(1-\rho)^{\mu_{Z}}}{(1-\rho)^{\mu_{T}}+\rho^{\mu_{T}}(1-\rho)^{\mu_{Z}}}$

In comparison to its marginal counterpart's Equation 14:

$\aleph_{Z}^{\rho}=\frac{(1-\rho)^{\mu_{Z}}}{\rho^{\mu_{Z}}+(1-\rho)^{\mu_{Z}}}$

And Equation 15:

$$
\aleph_{T}^{\rho}=\frac{\rho^{\mu_{T}}}{(1-\rho)^{\mu_{T}}+\rho^{\mu_{T}}}
$$

A sample counterpart of (12) is named observed logarithmic information nucleus and it is Equation 16:

$\ln \mathfrak{I}_{z, t} \approx(1-\rho) \bar{T}+\rho \bar{Z}$

Which could be exercised for testing a hypothesis about the parameter, $\rho$ since it is asymptotically normally distributed with mean (12) and variance Equation 17:

$$
\begin{aligned}
& \operatorname{Var}\left(\ln \mathfrak{I}_{z, t}\right) \\
& =\left\{\ln \left(\frac{1}{\rho}-1\right)\right\}^{2} \sigma_{T}^{2}+\{\ln (1-\rho)\}^{2} \sigma_{Z}^{2} \\
& -2 \sigma_{T} \sigma_{Z} \operatorname{Corr}(T, Z)\left\{\ln \left(\frac{1}{\rho}-1\right)\right\}\{\ln (1-\rho)\} \\
& \approx(n-1) \rho^{2}(1-\rho)^{2}
\end{aligned}
$$

Using the means (6.1) and (6.2), variances (6.3) and (6.4) and the correlation (9).

To test whether the estimate $\hat{\rho}_{\text {MLE }}$ in (10) is significant, we first formulate the null and research hypotheses respectively as $H_{0}: \rho=\hat{\rho}_{M L E}$ and $H_{1}: \rho>\hat{\rho}_{M L E}$. The p-value for the null hypothesis to be true is, after simplifications, that Equation 18:

$$
\begin{aligned}
& p \text {-value } \\
& \approx \operatorname{Pr}\left(Z>\left|\frac{\frac{\bar{t}}{\hat{\rho}_{M L E}}+\frac{\bar{z}}{\left(1-\hat{\rho}_{M L E}\right)}}{\sqrt{(n-1)}}-1\right|\right)
\end{aligned}
$$

The power is the probability of accepting a specified true alternative hypothesis $H_{1:} \rho=\rho_{*}$ and it is Equation 19:

$$
\begin{aligned}
& \text { power } \approx \operatorname{Pr}\left(|Z|<\left(1+z_{\alpha / 2}\right) a\right. \\
& \left\{\frac{\hat{\rho}_{M L E}\left(1-\hat{\rho}_{M L E}\right)}{\rho_{*}(1-\rho)}\right\} \\
& \left\{\frac{\left(1-\rho_{*}\right) \bar{t}+\rho_{*} \bar{z}}{\left(1-\hat{\rho}_{M L E}\right) \bar{t}+\hat{\rho}_{M L E} \bar{z}}\right\}-1
\end{aligned}
$$

\section{ILLUSTRATION}

In this section, the contents of the previous sections are illustrated using non adherence data in terms of the number of medicines prescribed of (Claxton, 2001) in Table 1. In this example, the prescription of medicines precedes the non-adherence. In the Group-1, a random sample of $n_{1}=50$ patients taking two or less number of prescribed medicines. In the Group-2, another independent random sample of $n_{2}=100$ patients taking three or more numbers of prescribed medicines are selected and enquired about their non-adherence to medicine in the survey. Thirty-seven in Group-1 and fifty-eight in Group-2 did not adhere to the prescribed medicines. The percent non-adherent in both groups is $\bar{t}=95 / 150=0.63$ and the percent adherent in both groups is $\bar{z}=55 / 150=0.36$. Using (9), the correlation between the adherence and non-adherence in this data is found to be -0.78 which is quite impressive. It means that if the number of non-adherent patients increase, then the number of adherent patients will decrease dramatically. Independent of the number of prescribed medicines, the probability for a patient to adhere the prescribed medicines is estimated to be $\hat{\rho}_{M L E}=0.22$ according to (10). Using (11), the probability estimate for a patient to be assigned two or less medicines is $\phi_{\mathrm{MLE}}=0.33$.

The p-value of $\hat{\rho}_{\text {MLE }}=0.22$ is about 0.23 according to (18) meaning that the estimate is not significant. Hence, the probability, $1-\hat{\rho}_{\text {MLE }}=0.78$ of non-adherence is highly significant. In an event, the true value of adherence is 0.7 (that is, $\rho_{*}=0.7$ ), the probability that the methodology of this article will accept is the power and it is 0.61 . The power is good. 
A consequence of not adhering to the prescribed medicines is the likelihood of hospitalization again as it is evident according to the data in Table 2 in the case of diabetic patients. In this illustration, not adhering to the prescribed medicines occurs first and the hospitalization again follows the non-adherence. The Group- 1 contains a random sample of $n_{1}=60$ diabetic patients whose hospitalization rate is less than $10 \%$ due to non-adherence of prescribed medicines. In the Group-2, another independent random sample of $n_{2}=$ 40 diabetic patients whose hospitalization rate is more than $10 \%$ due to non-adherence to prescribed medicines. Three in Group-1 and twenty-five in Group2 did not adhere to the prescribed medicines.

The percent non-adherent in both groups is $\bar{t}=28 / 100=0.28$ and the percent adherent in both groups is $\bar{z}=72 / 100=0.72$. Using (9), the correlation between the adherence and non-adherence in this data is found to be -0.44 which is not negligible. It means that if the number of non-adherent patients increase, then the number of adherent patients will decrease. Independent of the number of the hospitalization rate, the probability for a patient to adhere the prescribed medicines is estimated to be $\hat{\rho}_{M L E}=0.56$ according to (10). Using (11), the probability estimate for a patient to be hospitalized again is $\hat{\phi}_{M L E}=0.60$. The p-value of $\hat{\rho}_{M L E}=0.56$ is about 0.21 according to (18) meaning that the estimate is not significant. In an event, the true value of adherence is 0.7 (that is, $\rho_{*}=0.7$ ), the probability that the methodology of this article will accept is the power and it is 0.99 . The power is excellent.

Table 1. \# Medicines versus non adherence survey adherence survey

\begin{tabular}{|c|c|c|c|}
\hline \# prescribed medicines & Non adherent patients & Adherent patients & Sample size \\
\hline 2 or less & 37 & 13 & $n_{1}=50$ \\
\hline 3 or more & & 42 & $n_{2}=100$ \\
\hline Total & $\mathrm{T}=95$ & $Z=55$ & $\mathrm{n}=150$ \\
\hline$\hat{\rho}_{M L E}$ & $\hat{\rho}_{M L E}=\frac{(n-t)}{(n+t)}=0.22$ & & \\
\hline$\hat{\phi}_{M L E}$ & $\hat{\phi}_{M L E}=\frac{n_{1}}{\left(n_{1}+n_{2}\right)}=0.33$ & & \\
\hline Correlation between total adherent & $-\sqrt{(1-\rho)\left\{\frac{1}{n}+(1-\rho)\right\}}=-0.78$ & & \\
\hline $\begin{array}{l}\text { and non-adherent patients } \\
\text { p-value for } \hat{\rho}_{M L E}\end{array}$ & 0.23 & & \\
\hline Power for $H_{1}: \rho=0.7$ & 0.61 & & \\
\hline
\end{tabular}

Table 2. Hospitalization again among diabetic patients for not adhering to the prescribed medicines

\begin{tabular}{llll}
\hline Hospitalization rate & Non adherent patients & Adherent patients & Sample size \\
\hline Less than $10 \%$ & 3 & 57 & $n_{1}=60$ \\
$10 \%$ or more & 25 & 15 & $n_{2}=40$ \\
Total & $\mathrm{T}=28$ & $\mathrm{Z}=72$ & $\mathrm{n}=100$ \\
$\hat{\rho}_{M L E}$ & $\hat{\rho}_{M L E}=\frac{(n-t)}{(n+t)}=0.56$ & & \\
$\hat{\phi}_{M L E}$ & $\hat{\phi}_{M L E}=\frac{n_{1}}{\left(n_{1}+n_{2}\right)}=0.60$ & \\
Correlation between total & $-\sqrt{(1-\rho)\left\{\frac{1}{n}+(1-\rho)\right\}}=-0.44$ & \\
adherent and non-adherent patients & 0.21 & & \\
p-value for $\hat{\rho}_{M L E}$ & 0.99 & & \\
Power for $H_{1}: \rho=0.7$ & &
\end{tabular}




\section{CONCLUSION}

One of the concerns to the National Institute of Health and other healthcare agencies is the issue of patients' non-adherence to the prescribed medicines. Reasons for the patients' non-compliances are speculated and are verified only in some instances. The contents of this article are helpful to extract the data evidence about the patients' non-compliance to take the prescribed medicine and check the statistical significance of the data based estimates. The health insurance industries and the medical professionals would also benefit from the new statistical methodology of this article. In this era of globalized medical practices, the patients' non-adherence to the prescribed medicines is a vital factor to discuss to avoid legal law suits and promote the medical economy of nations around the world which play a prominent role.

\section{ACKNOWLEDGEMENT}

The researcher thanks and appreciates Mr. Kathir Selvan Shanmugam for bringing this topic and relevant articles to my attention.

\section{REFERENCES}

Ben-Naim, A., 2008. A Farewell to Entropy: Statistical Thermodynamics Based on Information. In: World Scientific Publishing Co, FL and U.S.A. ISBN-10: 9812707069.

Claxton, 2001. A systematic review of the associations between dose regimens and medication compliance. Clin. Therapeut., 23: 1296-1310.

Fisher, R., 1925. Theory of statistical estimation. Proc. Cambridge Philosophical Society, 22: 700-25.

DOI: $10.1017 / \mathrm{S} 0305004100009580$
Kullback, S. and R. Leibler, 1951. On information and sufficiency. Annals Math. Stat., 22: 79-86.

Shanmugam, R., 2013a. Informatics about fear to report rapes using bumped-up Poisson model. Am. J. Biostat., 3: 17-29. DOI: 10.3844/amjbsp.2013.17.29

Shanmugam, R., 2013b. Does over or under dispersion in inverse binomial data suggest anything? A case in point is the waiting time for both heart-lung transplants. Am. J. Biostat., 3: 30-37.

DOI: $10.3844 / \mathrm{amjbsp} .2013 .30 .37$

Shannon, C.A., 1948. Mathematical theory of communication. Bell Sys. Tech. J., 27: 379-423.

Stuart, A. and J. Ord, 1994. Kendall's Advanced Theory of Statistics, Distribution Theory. 1: Arnold Press, London.

Teamah, A.A.M. and A.M.T.E. Ahmed's, 2009. Random sum of mixtures of sum of bivariate exponential distributions. J. Math. Stat., 5: 270-275.

DOI: $10.3844 / \mathrm{jmssp} .2009 .270 .27$ 